\title{
Lung function response and side effects to rapamycin for lymphangioleiomyomatosis: a prospective national cohort study
}

\author{
Janet Bee, ${ }^{1}$ Sharon Fuller, ${ }^{1}$ Suzanne Miller, ${ }^{2}$ Simon R Johnson ${ }^{1,2}$
}

${ }^{1}$ National Centre for Lymphangioleiomyomatosis, Nottingham, UK

${ }^{2}$ Division of Respiratory Medicine, Nottingham Molecular Pathology Node and Nottingham Biomedical Research Centre, University of Nottingham, Nottingham, UK

\section{Correspondence to}

Professor Simon R Johnson, Division of Respiratory Medicine, University of Nottingham, Respiratory Medicine, Queens Medical Centre, Nottingham NG7 2UH, UK; simon.johnson@ nottingham.ac.uk

Received 7 August 2017 Revised 19 September 2017 Accepted 2 October 2017

Published Online First

9 October 2017

\section{Linked}

- http://dx.doi.org/10.1136/ thoraxjnl-2017-211135

Check for updates

To cite: Bee J, Fuller $S$ Miller $S$, et al. Thorax 2018:73:369-375

\section{ABSTRACT}

Rationale Mechanistic target of rapamycin

inhibitors reduce loss of lung function in

lymphangioleiomyomatosis (LAM), although their benefit varies between individuals. We examined lung function response and side effects to rapamycin in a national cohort.

Methods Subjects were receiving rapamycin for progressive lung disease. Clinical evaluation, detailed phenotyping, serial lung function, rapamycin and safety monitoring were performed according to a clinical protocol. Lung function change, measured as $\mathrm{FEV}_{1}$ slope $\left(\triangle \mathrm{FEV} \mathrm{V}_{1}\right)$, was reported for those treated for 1 year or longer.

Results Rapamycin was associated with improved $\triangle \mathrm{FEV}$, in 21 individuals where pretreatment data were available $(p<0.0001)$. In 47 treated for a mean duration of 35.8 months, mean $\triangle \mathrm{FEV}$, was +11 (SD 75) $\mathrm{mL} /$ year, although it varied from +254 to $-148 \mathrm{~mL}$ year. The quartile with the highest positive $\Delta \mathrm{FEV}$, had greater pretreatment $F E V_{1}(p=0.02)$ and shorter disease durations $(p=0.02)$ than the lowest quartile. Serum rapamycin level was positively associated with side effects $(p=0.02)$ but not $\triangle F E V$, over 1 year. Within the first month of therapy, apthous ulcers, nausea and diarrhoea were associated with higher rapamycin levels. Acne, oedema and menstrual irregularities tended to increase over the first year of therapy. At the end of observation, the prevalence of side effects was $5 \%$ or less.

Conclusions Rapamycin reduces lung function loss in LAM, although in some, $\triangle \mathrm{FEV}$, continues to fall at an accelerated rate. Poor response to rapamycin was associated with lower pretreatment lung function and longer disease duration but not serum level. Early intervention with low-dose rapamycin may preserve lung function and reduce side effects.

\section{INTRODUCTION}

Lymphangioleiomyomatosis (LAM) is a multisystem disease characterised by lung cysts, lymphatic abnormalities and a high prevalence of kidney angiomyolipomas. ${ }^{1}$ As a sporadic disease, LAM affects almost exclusively women with an incidence of 0.3 per million women per year: however, LAM is common in the autosomal genetic disease tuberous sclerosis complex (TSC-LAM) where it affects around half of adult women and some men. ${ }^{2}$ In LAM, cysts replace the lung parenchyma, resulting in recurrent pneumothorax and dyspnoea, which

\section{Key messages}

What is the key question?

- How can we best optimise the riskbenefit balance of mechanistic target of rapamycin (mTOR) inhibition for loss of lung function in individuals with lymphangioleiomyomatosis (LAM)?

What is the bottom line?

- Loss of lung function may continue in some individuals treated with rapamycin, and a poor response to the drug is more likely in those with lower lung function and longer disease duration at the start of treatment. Lower levels of rapamycin are associated with fewer side effects but equal benefit compared with higher levels.

Why read on?

- We have used a prospective national cohort study to understand the relationship between rapamycin levels, lung function response and side effects to improve the use of mTOR inhibitors in women with LAM.

can progress over a variable period of years to result in respiratory failure. Lymphatic involvement can cause lymphatic masses and chylous collections in the thorax and abdomen. Angiomyolipomas are frequently small and asymptomatic but may enlarge and bleed, necessitating urgent treatment.

The lungs and lymphatics of women with LAM are infiltrated by LAM cells: a clone of cells expressing markers of both melanogenesis and smooth muscle differentiation. LAM cells in both sporadic and TSC-LAM have bi-allelic inactivating mutations in either the TSC- 1 or more frequently the TSC- 2 gene. ${ }^{3}$ Loss of function of TSC- $1 / 2$ leads to constitutive activation of the mechanistic target of rapamycin (mTOR), resulting in altered translation of $5^{\prime}$ cap-dependent genes, uncontrolled proliferation, dependence on autophagy and a metabolic shift toward energy generation by glycolysis. ${ }^{4}$ Suppression of mTOR signalling in women with LAM is associated with reduced loss of lung function, ${ }^{5-7}$ reduction in angiomyolipoma volume $e^{8-10}$ and reduced chylous complications. ${ }^{11}$ The use of mTOR inhibitors has become the standard of care for progressive lung disease in sporadic LAM ${ }^{12}$ and 
in TSC for LAM, angiomyolipoma and subependymal giant cell astrocytoma. $^{13}$

Although mTOR inhibitors reduce the rate of loss of $\mathrm{FEV}_{1}$ in those with progressive disease, some of those treated continue to decline, and it is not clear how to manage these individuals. ${ }^{14} 15$ Further, mTOR inhibition is associated with oral mucositis, nausea, diarrhoea, oedema, acne, hyperlipidaemia, proteinuria, pneumonitis and a theoretical risk of future malignancy. ${ }^{14}$ As many women with LAM develop progressive disease in their $30 \mathrm{~s}$, the potential cumulative exposure to mTOR inhibitors over decades means that the risk of adverse effects is significant. Although data from clinical trials and selected populations have reported clinical response and side effects, ${ }^{716}$ how these are related to drug levels has not been examined. As LAM is an orphan disease with no surrogate markers of disease activity, performing conventional randomised studies to understand different treatment regimens requires international collaborations over many years at potentially prohibitive cost. To address how to best preserve lung function, limit side effects from mTOR inhibition and inform future clinical trial design, we used a prospective national cohort study of women with LAM treated with rapamycin for progressive lung disease over a period of 1-6years study to examine the relationship between lung function response, side effect profile and clinical features.

\section{METHODS}

The study was conducted at the National Centre for Lymphangioleiomyomatosis at Nottingham University Hospitals NHS Trust between 2011 and 2017. The Centre is funded by highly specialised commissioning for NHS England to provide a comprehensive clinical service for women with LAM in the UK. Women receiving care at the LAM Centre are invited to participate in an observational cohort study. All participants gave written informed consent.

The diagnosis of LAM was made according to American Thoracic Society (ATS) criteria. ${ }^{12}$ Clinical history, lung function, imaging and blood samples were collected at recruitment. Duration of disease was calculated as the interval between the first symptom attributable to LAM (eg, first pneumothorax or onset of dyspnoea) and the start of treatment with rapamycin.

All subjects had progressive lung disease. The decision to start rapamycin was made on clinical grounds alone. Subjects were treated according to a standard clinical protocol: individuals had baseline full blood count, urea and electrolytes, liver function tests, lipid profile, urine protein:creatinine ratio (PCR) and a pregnancy test. All were counselled on the indication for treatment, need for monitoring, potential adverse effects and drug interactions. Advice was given around intercurrent illness, surgery, pregnancy and supported with written information. All were given contact details of the Centre nurse specialist for queries and advice.

Rapamycin, generally 1 or $2 \mathrm{mg}$ orally once daily, was prescribed and a trough level measured 10-14 days later. One month later, subjects returned for a clinical assessment of side effects, a trough rapamycin level, full blood count, urea and electrolytes, liver function tests, lipid profile and urine PCR and the dose adjusted on the basis of serum level or side effects if required. After 3 months, these tests were repeated and $\mathrm{FEV}_{1}$ and $\mathrm{DL}_{\mathrm{CO}}$ measured. This assessment was repeated at 6 months of treatment and every 6 months thereafter. Subjects saw both a consultant physician (SJ) and nurse specialist (SF) at each visit, and side effects were recorded.
Lung function was measured according to ATS/European Respiratory Society standards ${ }^{17}$ with predicted values calculated using regression equations from Quanjer et al. ${ }^{18}$ Change in $\mathrm{FEV}_{1}$ over time $\left(\Delta \mathrm{FEV}_{1}\right)$ was calculated as the regression slope of all $\mathrm{FEV}_{1}$ measurements taken either before rapamycin or during treatment using Microsoft Excel as described. ${ }^{15}{ }^{19}$ As shorter periods of observation and fewer individual measurements are associated with variation away from the group mean for $\Delta \mathrm{FEV}_{1}$ in those with $\mathrm{LAM}^{19}{ }^{19} \Delta \mathrm{FEV}_{1}$ was only reported for periods of observation of 1 year or greater. Serum vascular endothelial growth factor-D (VEGF-D) was measured using a human quantikine VEGF-D ELISA (R\&D Systems, UK). Serum cholesterol, urea and electrolytes, full blood count, liver function and urine PCR were measured in the clinical service laboratory at Nottingham University NHS Trust.

Data were analysed in GraphPad Prism V.7.03 (GraphPad Software, La Jolla, California, USA), MS Excel and SPSS V.24 (IBM). Data were tested for normality using the D'Agostino-Pearson test. Paired $\triangle \mathrm{FEV}_{1}$ values before and after therapy were compared by paired Student's t-test, comparison of cholesterol and PCR before and after treatment by paired Student's t-test for the individual subjects, non-parametric data by Fischer's exact and $\chi^{2}$ tests and association of rapamycin level with side effects and laboratory markers by Pearson correlation. Student's t-tests were reported uncorrected unless stated. Comparison of pretreatment disease duration and lung function, drug level and phenotype characteristics with $\triangle \mathrm{FEV}_{1}$ was performed for the quartiles with the best and poorest response to rapamycin by unpaired Student's t-tests and for the group as a whole by multivariable analysis. $\mathrm{p}$ Values of $<0.05$ were considered significant.

\section{RESULTS}

\section{Study cohort}

Forty-seven women with definite LAM defined by ATS criteria and progressive lung disease who had been treated with rapamycin for longer than 1 year were included in the study. The mean age at the start of treatment was 43.6 (SD 8.2) years and the mean duration of treatment was 35.8 (SD 18) months equating to 143 patient/years of treatment observation. Mean pretreatment $\mathrm{FEV}_{1}$ was $52 \%$ (19) and $\mathrm{DL}_{\mathrm{CO}} 42 \%$ (12) of the predicted values. Nine (19\%) had TSC, 18 (38\%) had a history of pneumothorax, angiomyolipoma was present in 9 (19\%) and lymphatic complications, defined as chylous collections or lymphatic masses visible on CT scanning, were also present in 9 (19\%) individuals.

\section{Effect of rapamycin on $\triangle \mathrm{FEV}$}

Twenty-one of the 47 individuals also had more than 1 year of observation of lung function prior to treatment with rapamycin. The remaining 26 had been treated elsewhere or required treatment at diagnosis and pretreatment data were not available. There was no significant difference in age, baseline lung function or response lung function response to rapamycin within those with and without pretreatment data. Within these 21, the mean duration of observation was 38 (SD 22) and 36 (SD 20) months pretreatement and post-treatment, respectively. Mean pretreatment $\Delta \mathrm{FEV}_{1}$ was $-150 \mathrm{~mL} /$ year $(95 \% \mathrm{CI}-220$ to -79$)$ and during treatment was $+35 \mathrm{~mL} /$ year $(95 \% \mathrm{CI}+9$ to +61 , $\mathrm{p}<0.0001)$. Furthermore, loss of $\mathrm{FEV}_{1}$ improved during rapamycin treatment in all individuals (figure 1A).

For all 47 subjects, mean treatment $\Delta \mathrm{FEV}_{1}$ was $+11(\mathrm{SD} 75) \mathrm{mL} /$ year, although between individuals, $\Delta \mathrm{FEV}_{1}$ varied from +254 to $-148 \mathrm{~mL} /$ year. To understand the characteristics associated with 

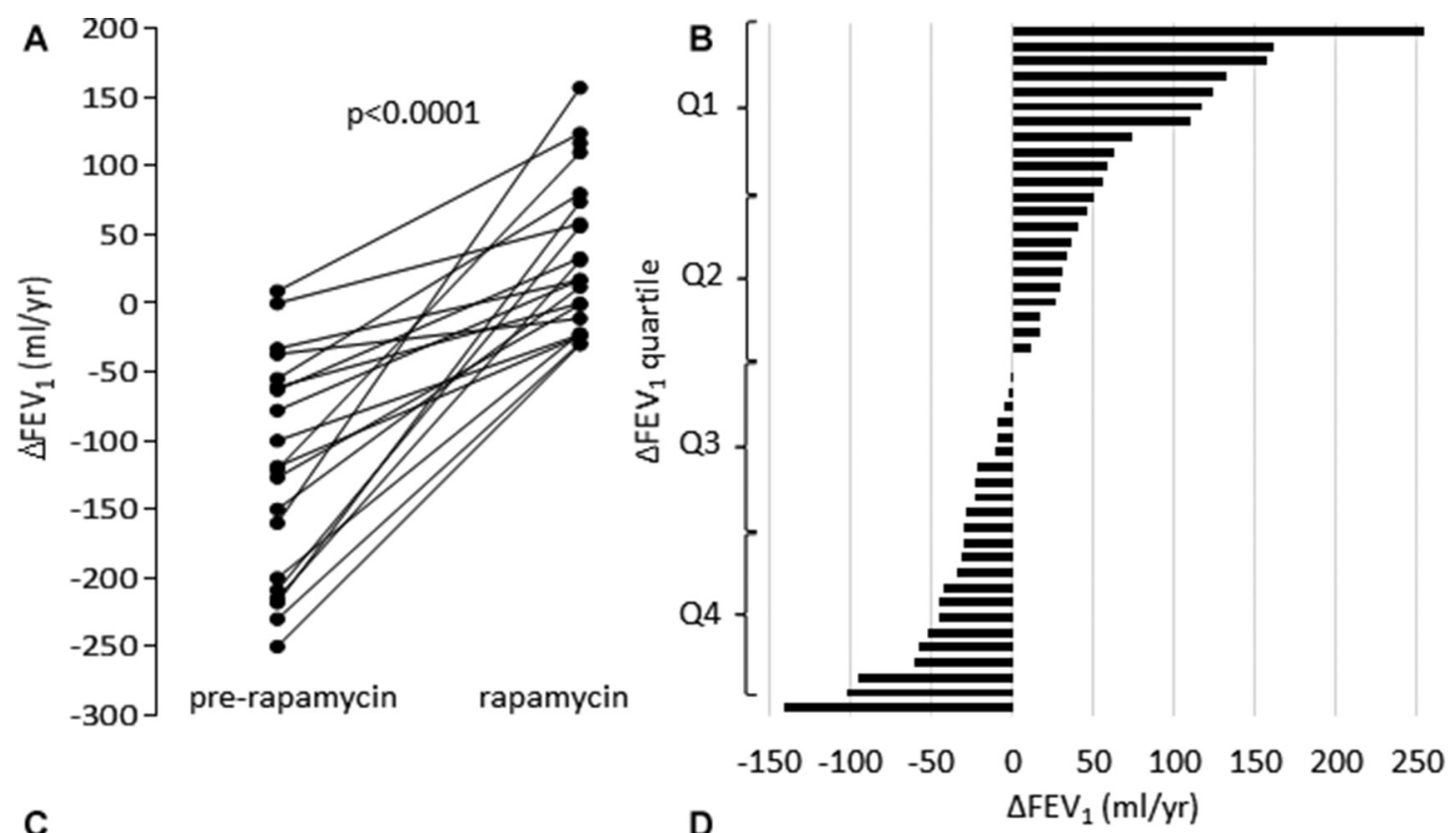

C

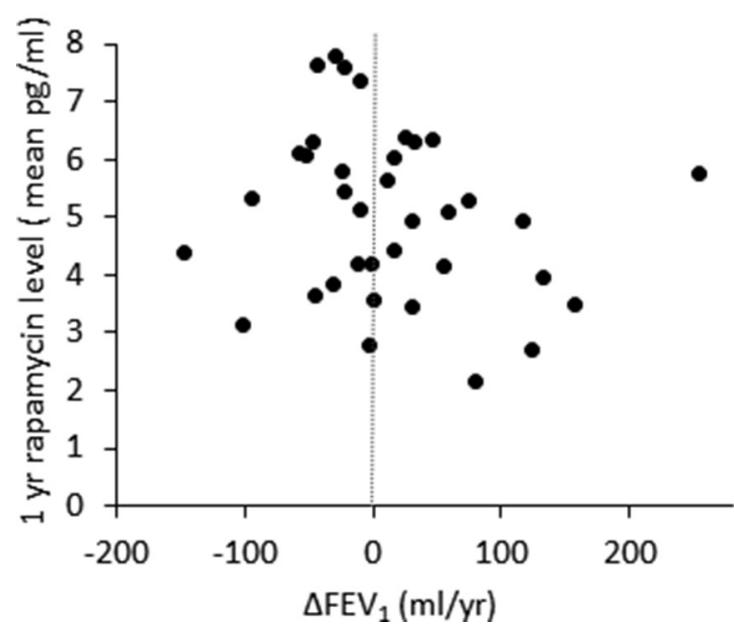

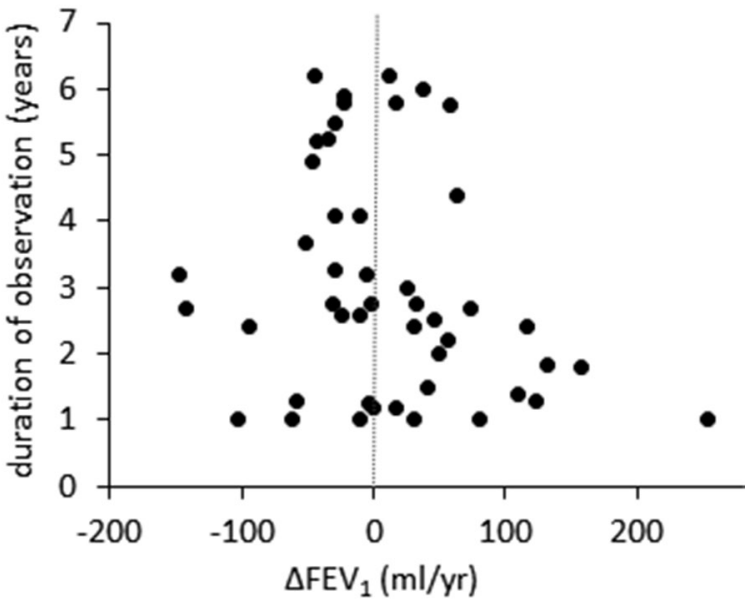

Figure 1 Change in FEV in response to rapamycin. (A) Loss of FEV1 ( $\triangle \mathrm{FEV} 1)$ in individuals prior to and during treatment with rapamycin. $\triangle \mathrm{FEV} 1$ improved in all of those treated and was significant in the group as a whole. (B) Variation in $\triangle F E V 1$ between 47 individuals treated with rapamycin. (C) Lung function response to rapamycin $(\triangle \mathrm{FEV} 1)$ is not related to mean serum rapamycin level over one1 year. (D) Relationship between $\triangle \mathrm{FEV} 1$ and duration of rapamycin treatment. Outlying values of $\triangle \mathrm{FEV} 1$ tend to be associated with shorter durations of observation.

differing responses to rapamycin, subjects were analysed in two ways. First, subjects were divided into quartiles according to their treatment $\triangle \mathrm{FEV}_{1}$ (figure $1 \mathrm{~B}$ ). The $25 \%$ of those with the highest positive $\triangle \mathrm{FEV}_{1}$ when treated with rapamycin (quartile 1) had significantly higher pretreatment $\mathrm{FEV}_{1}$ and shorter disease duration than the $25 \%$ of those with the lowest negative $\triangle \mathrm{FEV}_{1}$ (quartile 4). There was no significant association with serum rapamycin level (figure 1C), the presence of angiomyolipoma, pneumothorax or lymphatic complications (table 1). Those with the highest or lowest values of $\Delta \mathrm{FEV}_{1}$ tended to have periods of observation of less than 3 years (figure 1D). Next, the relationship between $\triangle \mathrm{FEV}_{1}$ and pretreatment disease duration, pretreatment $\mathrm{FEV}_{1}$ and $\mathrm{DL}_{\mathrm{CO}}$ was subjected to multivariate analysis for all subjects. In initial univariate analyses, disease duration (unadjusted $\mathrm{p}=0.02$ ) and baseline $\mathrm{FEV}_{1}$ (unadjusted $\mathrm{p}=0.006$ ) were associated with treatment response, although baseline $\mathrm{DL}_{\mathrm{CO}}$ was not (unadjusted $p=0.33$ ). Neither disease duration nor baseline $\mathrm{FEV}_{1}$ remained significant in a subsequent multivariate analysis.
To determine if the lack of association between serum rapamycin levels and treatment response was due to maintaining higher serum drug levels in those with more severe disease, we examined the association between rapamycin level and baseline lung function. There was a slight negative correlation between rapamycin level over 1 year and baseline $\mathrm{FEV}_{1}(\mathrm{r}=-0.37(95 \%$ $\mathrm{CI}-0.62$ to -0.048$), \mathrm{p}=0.026)$ but not $\mathrm{DL}_{\mathrm{CO}}(\mathrm{r}=-0.28(95 \%$ $\mathrm{CI}-0.56$ to 0.054$), \mathrm{p}=0.099)$.

\section{Side effects of rapamycin}

Due to follow-up schedules, intercurrent illness and travel issues to the LAM centre, 11 subjects had some missing values for rapamycin levels or side effects, and these subjects were excluded from the side effect profile analysis. Most individuals experienced side effects, which required a dose reduction in $25 \%$ of cases, although all continued with treatment. The most common side effects were apthous ulcers, nausea, acne, oedema and menstrual irregularities (table 2). Within the first month of therapy, 
Table 1 Clinical characteristics of quartiles of $\triangle \mathrm{FEV} 1$ response to rapamycin

\begin{tabular}{|c|c|c|c|c|c|}
\hline$\Delta \mathrm{FEV}_{1}$ response quartile & Q1 & Q2 & Q3 & Q4 & Q1 vs Q4 \\
\hline n & 11 & 12 & 12 & 12 & (p) \\
\hline \multicolumn{6}{|l|}{ Rapamycin-treated $\Delta \mathrm{FEV}_{1}$} \\
\hline Range (mL/year) & 254 to 56 & 50 to 0 & -1 to -30 & -31 to -148 & - \\
\hline Mean (mL/year) & $111(58)$ & $28(15)$ & $-17(11)$ & $-71(41)$ & $<0.0001$ \\
\hline Duration of treatment (years) & $2.4(1.3)$ & $3.4(1.6)$ & $2.8(1.1)$ & $2.4(2.1)$ & 0.09 \\
\hline \multicolumn{6}{|c|}{ Clinical characteristics at start of treatment } \\
\hline Pretreatment $\Delta \mathrm{FEV}_{1}\left(\mathrm{~mL} /\right.$ year) ${ }^{*}$ & $-186(238)$ & $-100(69)$ & $-152(77)$ & $-53(202)$ & 0.20 \\
\hline Age (years) & $41(9)$ & $45(11)$ & $44(8)$ & $45(6)$ & 0.13 \\
\hline Disease duration (years) & $6.1(5.3)$ & $13.0(10.2)$ & $8.2(5.0)$ & $11.3(5.9)$ & 0.02 \\
\hline $\mathrm{FEV}_{1}(\%$ predicted $)$ & $67(23)$ & $42(10)$ & $52(17)$ & $48(20)$ & 0.02 \\
\hline $\mathrm{DL}_{\mathrm{co}}(\%$ predicted $)$ & $46(11)$ & $40(10)$ & $45(14)$ & $40(15)$ & 0.13 \\
\hline \multicolumn{6}{|l|}{ Disease features present } \\
\hline Angiomyolipoma & 6 & 5 & 6 & 3 & NS \\
\hline Lymphatic disease & 3 & 3 & 1 & 2 & NS \\
\hline TSC & 3 & 2 & 2 & 2 & NS \\
\hline Ever had pneumothorax & 5 & 4 & 6 & 3 & NS \\
\hline \multicolumn{6}{|l|}{ Post-treatment laboratory values } \\
\hline Trough rapamycin level $(\mathrm{ng} / \mathrm{mL}) \dagger$ & $4.2(1.2)$ & $5.2(1.2)$ & $5.6(1.7)$ & $5.2(1.5)$ & 0.07 \\
\hline Urine protein:creatinine ratio $\ddagger$ & $8(5-366)$ & $9(6-43)$ & $11(5-79)$ & $8(6-15)$ & 0.84 \\
\hline
\end{tabular}

${ }^{*}$ For 21 subjects only.

tMean value over 12 months ( $p$ for Student's t-test).

$\ddagger$ Median (range) value after 12 months ( $p$ for Mann-Whitney test). NS for non-parametric data analysed by $\chi^{2}$ and Fischer's exact test.

Table 2 Side effects experienced while taking rapamycin according to duration of treatment and categorised by the Common Terminology Criteria for Adverse Events

\begin{tabular}{|c|c|c|c|c|c|}
\hline & $\%$ a & ted & & & \\
\hline Treatment duration (months) & 1 & 6 & 12 & Latest & Ever \\
\hline Common Terminology Criteria for $\mathrm{Ac}$ & verse & nts $C$ & ory & & \\
\hline Gastrointestinal & & & & & \\
\hline Oral mucositis & 58 & 35 & 24 & 13 & 65 \\
\hline Diarrhoea & 32 & 12 & 10 & 0 & 41 \\
\hline Nausea & 24 & 3 & 5 & 0 & 24 \\
\hline Abdominal pain & 6 & 0 & 0 & 0 & 6 \\
\hline Indigestion & 6 & 0 & 0 & 0 & 6 \\
\hline Bloating & 0 & 3 & 5 & 6 & 9 \\
\hline Reflux symptoms & 0 & 0 & 0 & 5 & 5 \\
\hline Dermatological & & & & & \\
\hline Acne & 6 & 17 & 18 & 0 & 41 \\
\hline Rash & 3 & 10 & 0 & 0 & 12 \\
\hline Dry skin & 6 & 0 & 0 & 0 & 6 \\
\hline Soft tissues & 5 & 7 & & & \\
\hline Peripheral oedema & & & 0 & 0 & 9 \\
\hline $\begin{array}{l}\text { Sexual or reproductive function } \\
\text { Menstrual cycle disturbances }\end{array}$ & 0 & 7 & 5 & 0 & 15 \\
\hline Constitutional symptoms & & & & & \\
\hline Fatigue & 3 & 7 & 0 & 5 & 9 \\
\hline Infection & & & & & \\
\hline Upper respiratory/bronchitis & 12 & 24 & 10 & 13 & 35 \\
\hline Tonsillitis & 3 & 7 & 0 & 0 & 6 \\
\hline Pneumonia & 0 & 3 & 0 & 5 & 5 \\
\hline Urinary tract infection & 0 & 7 & 0 & 0 & 6 \\
\hline Skin infection & 3 & 7 & 0 & 0 & 9 \\
\hline Musculoskeletal & & & & & \\
\hline Arthralgia or myalgia & 3 & 3 & 5 & 0 & 6 \\
\hline
\end{tabular}

apthous ulcers, nausea and diarrhoea were most common. These fell from 59\%, 32\% and 24\% after 1 month to $15 \%, 7 \%$ and $2 \%$, respectively, after 1 year of therapy (figure $2 \mathrm{~A}$ ). The incidence of acne, oedema and menstrual irregularities tended to increase over the first year of therapy from 7\%, 5\% and $0 \%$, respectively, at 1 month to $17 \%, 12 \%$ and $7 \%$ of individuals, respectively, at 12 months. By the end of the observation period (mean treatment duration of 35.8 months), the prevalence of all side effects was $5 \%$ or less in all those treated. Pneumonitis was not observed at any time.

\section{Relationship between side effects and rapamycin level}

We next examined the association between serum rapamycin level and the six most common side effects over the first month and first 12 months of treatment. After 1 month, 40\% of those with a rapamycin level $<3 \mathrm{pg} / \mathrm{mL}$ developed apthous ulcers but none of the other six common side effects. During the first month of treatment, apthous ulcers and diarrhoea were associated with increasing rapamycin levels (figure 2B). An increasing incidence of apthous ulcers and diarrhoea was also associated with higher mean serum rapamycin levels over 1 year, as were nausea, acne and menstrual irregularities (figure 2C). While the prevalence of individual side effects for differing rapamycin levels did not allow statistical analysis, the total occurrence of the six major side effects was positively correlated with mean rapamycin level over 1 year $(r=0.39, \mathrm{p}=0.02)$.

\section{Effect of rapamycin on laboratory values}

Treatment with rapamycin was associated with a significant elevation of serum cholesterol within the first month of treatment from a baseline of $5.1 \mathrm{mmol} / \mathrm{L}(95 \% \mathrm{CI} 4.8$ to 5.4$)$ to 5.6 (95\% CI 5.2 to 6.0 ), which was sustained for the whole 


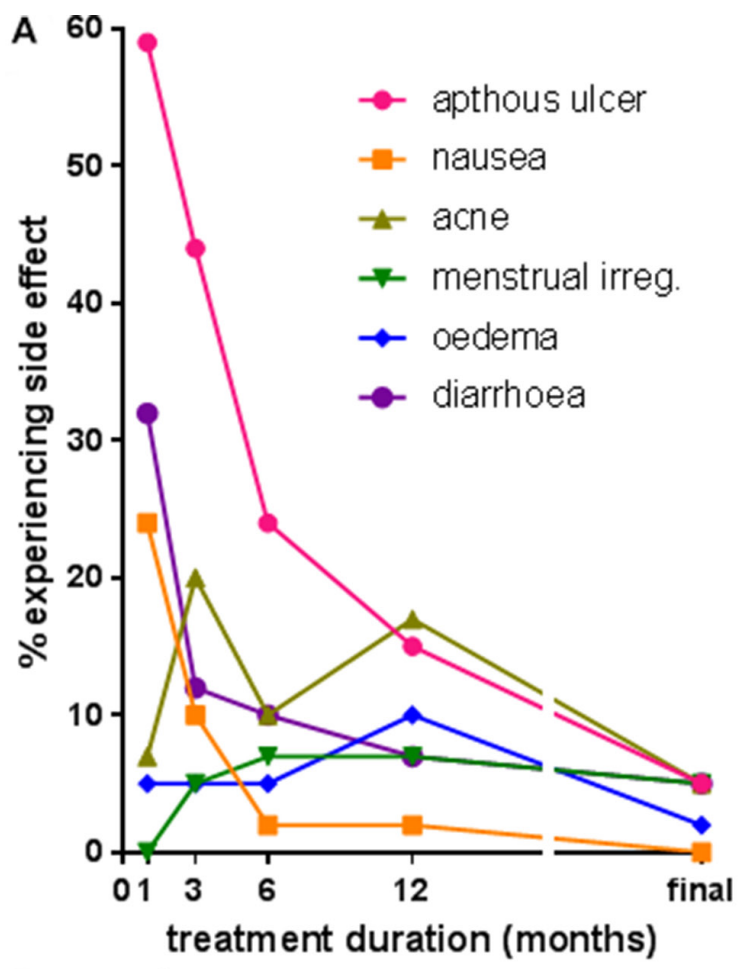

B 1 month

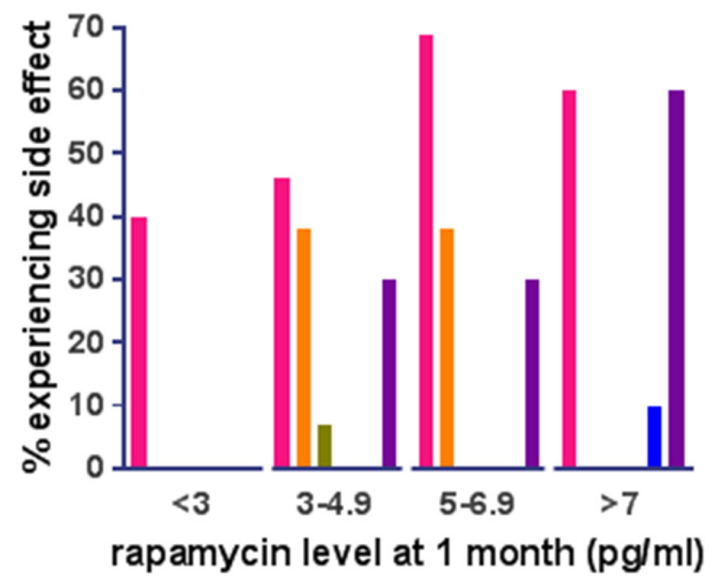

C 1 year

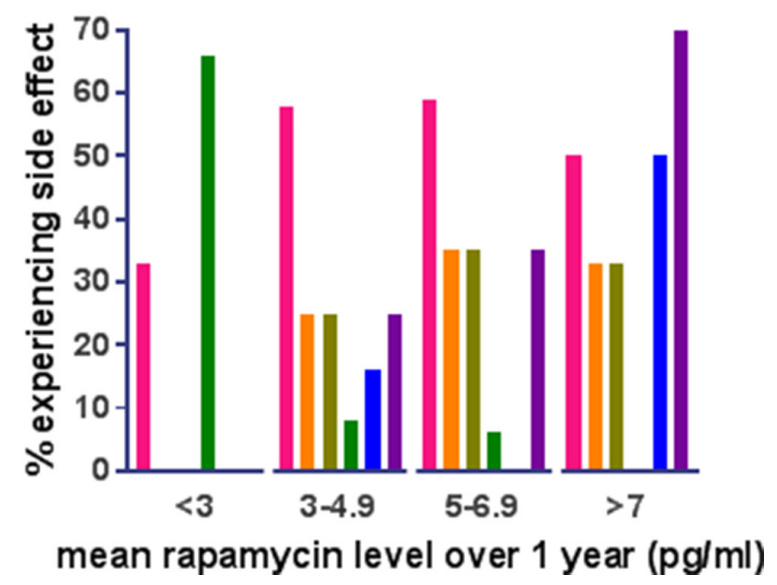

Figure 2 Side effects of rapamycin treatment. (A) Relationship between side effects and treatment duration. Colours of markers and side effects are consistent in subsequent figures. (B) Side effects according to rapamycin level after 1 month of therapy. (C) Side effects according to rapamycin level after 1 year. duration of treatment (figure 3A). Urinary PCR was variable between individuals, and although there was a trend toward an increase PCR over the first year of treatment, this returned to pretreatment values over the course of treatment (mean duration 35 months; figure $3 \mathrm{~A}$ ). There were no significant changes in haemoglobin and renal or liver function over the treatment period (data not shown).

Although those with higher plasma rapamycin levels tended to have the larger elevations of serum cholesterol, the serum level of rapamycin over 12 months of treatment was not significantly related to the increase in either cholesterol or urine PCR (figure 3B).

\section{DISCUSSION}

We have examined the lung function response to rapamycin and related side effects in a prospective national cohort of women with progressive lung disease due to LAM. Overall, rapamycin was associated with reduced disease progression, but a significant minority continued to lose lung function at an accelerated rate. While side effects were common, particularly at higher doses of rapamycin, they did not require cessation of therapy and remained at tolerable levels over several years. Likewise, elevations in serum cholesterol and proteinuria were small and manageable.

In all cases, treatment with rapamycin was associated with improvements in $\triangle \mathrm{FEV}_{1}$. In this study, the mean $\triangle \mathrm{FEV}_{1}$ observed for almost 3 years of treatment was $+11 \mathrm{~mL} /$ year and similar to that observed over shorter periods and in other cohorts. ${ }^{514}$ What is striking is the variability in post-treatment $\Delta \mathrm{FEV}_{1}$, which, although observed in other studies, has not been previously addressed. Those with the most favourable values of $\triangle \mathrm{FEV}_{1}$ tended to have had LAM for less time and had better lung function, particularly $\mathrm{FEV}_{1}$, at the start of treatment. Importantly, this favourable lung function response was not simply accounted for by higher rapamycin levels in this group. It is possible that those with the least response to rapamycin have disease that is less dependent on mTOR dysregulation as suggested by the finding that signalling pathways other than mTOR contribute to tumourigenesis in LAM cells. ${ }^{20}$ In addition, VEGF-D is synthesised by LAM cells and is a transcriptional target of mTOR: the finding that higher levels of serum VEGF-D are associated with a better response to rapamycin is potentially consistent with differing levels of mTOR dysregulation and influence on the disease phenotype between individuals. ${ }^{21}$ Conversely, resistance to rapamycin may be acquired over time as occurs in breast cancer cells in vitro $^{22}$ : with potential mechanisms including cellular efflux of rapamycin by ATP-binding cassette transporters, mutations in rapamycin-binding proteins, including FKBP, or loss of function or feedback in pathways such as Akt and retinoblastoma protein mediating some rapamycin-dependent growth effects. ${ }^{23} 24$ It is also possible that, in more advanced disease, LAM-related tissue remodelling itself, including recruitment of wild-type cells, can support LAM nodule growth and lung destruction, independent of mTOR signalling. ${ }^{25}$ LAM is variable both in severity and its clinical manifestations, and it is conceivable that specific LAM phenotypes or groups of clinical features may be associated with favourable or unfavourable responses to rapamycin. The finding that those with the most and least favourable responses had different pretreatment characteristics but that these characteristics were not significant in across the group as a whole suggests that those with good or poor responses to mTOR inhibition have different phenotypes or drug sensitivity rather than all patients being part of a continuous 
A
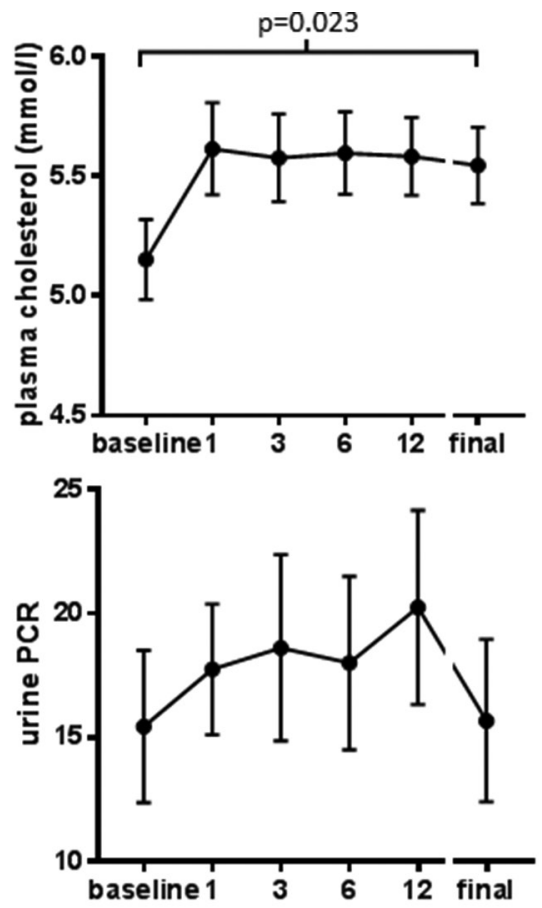

duration of treatment (months)
B
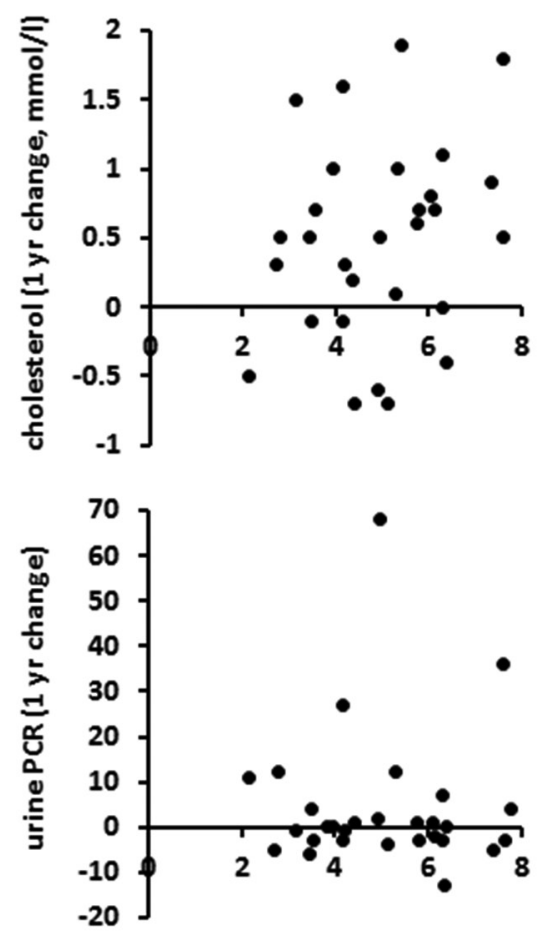

mean 1 year rapamycin level (pg/ml)

Figure 3 Effect of rapamycin on serum cholesterol and urine protein. (A) Mean and SE of plasma cholesterol and urine PCR during treatment. (B) Association of serum rapamycin level with serum cholesterol and urine PCR after 1 year of therapy. PCR, protein:creatinine ratio.

spectrum of rapamycin responsiveness, although larger prospective studies will be required to address this point definitively. Previous reports have suggested that those with chylous complications respond well to rapamycin, ${ }^{11}$ and although in our study, those with positive values of $\triangle \mathrm{FEV}_{1}$ had more lymphatic disease than those with negative $\Delta \mathrm{FEV}_{1}$ values, this was not significantly different overall. It is possible that the benefit obtained from rapamycin in lymphatic remodelling leading to reductions in lymphatic masses and chylous collections does not translate into longer-term effects on lung parenchymal damage. However, our study was not powered to definitively assess the association of individual or groups of clinical features and treatment response.

We were interested to observe that there was no association between $\triangle \mathrm{FEV}_{1}$ and serum rapamycin level. Although there was a weak association between serum rapamycin level and lower $\mathrm{FEV}_{1}$ at the start of therapy, we cannot completely exclude the possibility that the lack of a dose-response relationship was due to this, although the level of difference is unlikely to have influenced the findings significantly. Our findings would be consistent with the idea that serum levels greater than $2 \mathrm{pg} / \mathrm{mL}$ are sufficient to treat progressive lung disease in most women with LAM. While most clinical trials of rapamycin showing benefits on lung function and angiomyolipoma have titrated serum levels to $5-10 \mathrm{pg} / \mathrm{mL}$, a retrospective study has suggested that lower serum rapamycin levels may be equally effective. ${ }^{26}$ Our study, examining the group as a whole, was unable to assess if there was a dose-response relationship between rapamycin level and $\Delta \mathrm{FEV}_{1}$ for individual subjects; however, this study would require very long periods of observation and has not yet been performed.

Side effects were common and tended to occur either at the start of treatment and reduce over time (mouth ulcers, nausea and diarrhoea) or some weeks into treatment and persist (acne, oedema, menstrual disturbance). In excess of 1 year of treatment, individual side effects occurred in less than $5 \%$ of individuals overall. Unlike lung function response, there was a relationship between side effects and serum rapamycin levels with mean serum level positively associated with the six most common side effects over 1 year with nausea and diarrhoea particularly increasing at higher serum levels. Changes in laboratory indices mainly comprised a persistent elevation in serum cholesterol and transient proteinuria but in no individuals resulted in the need to stop therapy. There was no clear correlation between rapamycin level and elevation in either urinary protein or serum cholesterol.

Our study was a real-world observational cohort of those receiving rapamycin for progressive lung disease. As the Centre provides care for over two-thirds of the UK population with a range of ages, clinical phenotypes and severity, the study benefited from being an unselected group representative of the disease as a whole. ${ }^{15}$ Subjects were treated according to a standard protocol and seen at all visits by the same clinical team and had lung function measured at the same centre. However, various limitations are inherent real-world cohorts, side effect reporting relied on patient recall and treatment duration was not the same for all participants. As a consequence, extremes of $\Delta \mathrm{FEV}_{1}$ where values exceeded $100 \mathrm{~mL} /$ year in either direction tended to be those with a duration of observation of less than 3 years, suggesting that some of this variability may be attributed to shorter-term variation or regression to the mean. ${ }^{19}$ However, even in those treated for longer periods, there was still significant variability in response. As rapamycin has become the standard of care from those with progressive lung disease, it is no longer possible to study untreated control subjects matched for disease severity. However, these subjects had similar overall levels of base line lung function, lung function response and side effects seen in other studies including the Multicenter International Lymphangioleiomyomatosis Efficacy of Sirolimus (MILES) trial, a 1-year randomised placebo controlled trial of rapamycin. ${ }^{514}$ 
Although serum rapamycin level and baseline lung function were not examined in the MILES trial, MILES has been the only other study to examine variability in response to rapamycin where a poorer response over 1 year was associated with low baseline serum VEGF-D values, perhaps consistent with less-mTOR-dependent disease. $^{521}$

Our findings have clinical implications and suggest that lower serum rapamycin levels are associated with fewer side effects but no reduction in efficacy with potential benefits in reduced longterm toxicity and costs. The observation that better responses were associated with earlier disease is consistent with the idea that early intervention with low-dose rapamycin may be the best way to preserve lung function. The decision on when to offer treatment with rapamycin to balance preservation of lung function against cumulative side effects, and indeed the risk of unnecessary treatment of some individuals, is a critical issue for physicians and women with LAM. The recent ATS/Japanese Respiratory Society statement recommends the introduction of treatment for those with $\mathrm{FEV}_{1}$ or $\mathrm{DL}_{\mathrm{CO}}<70 \%$ of the predicted value. ${ }^{12}$ It is currently not known how best to identify those with active disease earlier in their natural history. Those with active disease may include those with falling $\mathrm{FEV}_{1}$ while still in the normal range, those with normal spirometry but impaired $\mathrm{DL}_{\mathrm{CO}}$ or exertional hypoxaemia. In addition, higher levels of VEGF-D and younger age at presentation are more likely to indicate active disease and benefit from early treatment. The use of lower doses of rapamycin than previously studied with the aim of preserving lung function in early disease is to be tested in a randomised clinical trial (ClinicalTrials.gov Identifier: NCT03150914). It is also important to recognise that some treated with rapamycin continue to lose lung function at an accelerated rate, and a formal study of disease phenotypes associated with poor lung function response and a protocol considering adjunctive therapy to rapamycin would improve management of individuals with aggressive and less mTOR inhibitor responsive disease, although this would require international collaboration.

Contributors SRJ conceived the study. SRJ and SF saw all participants. JB, SF, SM and SRJ recorded and analysed the data. All authors contributed to the final manuscript.

\section{Competing interests None declared.}

Patient consent Detail has been removed from this case description/these case descriptions to ensure anonymity. The editors and reviewers have seen the detailed information available and are satisfied that the information backs up the case the authors are making.

Ethics approval East Midlands Research Ethics Committee (13/EM/0264).

Provenance and peer review Not commissioned; externally peer reviewed.

(c) Article author(s) (or their employer(s) unless otherwise stated in the text of the article) 2018. All rights reserved. No commercial use is permitted unless otherwise expressly granted.

\section{REFERENCES}

1 Johnson SR, Taveira-DaSilva AM, Moss J. Lymphangioleiomyomatosis. Clin Chest Med 2016;37:389-403.
2 Harknett EC, Chang WYC, Byrnes S, et al. Regional and national variability suggests underestimation of prevalence of Lymphangioleiomyomatosis. Q J Med 2011;104:971-9

3 Finlay G. The LAM cell: what is it, where does it come from, and why does it grow? Am J Physiol Lung Cell Mol Physiol 2004;286:690L-3.

4 Lam HC, Nijmeh J, Henske EP. New developments in the genetics and pathogenesis of tumours in tuberous sclerosis complex.J Pathol 2017;241:219-25.

5 McCormack FX, Inoue Y, Moss J, et al. Efficacy and safety of sirolimus in lymphangioleiomyomatosis. N Engl J Med 2011;364:1595-606.

6 Goldberg HJ, Harari S, Cottin V, et al. Everolimus for the treatment of lymphangioleiomyomatosis: a phase II study. Eur Respir J 2015;46:783-94.

7 Takada T, Mikami A, Kitamura N, et al. Efficacy and safety of long-term Sirolimus therapy for Asian patients with Lymphangioleiomyomatosis. Ann Am Thorac Soc 2016;13:1912-22.

8 Bissler JJ, McCormack FX, Young LR, et al. Sirolimus for angiomyolipoma in tuberous sclerosis complex or lymphangioleiomyomatosis. N Engl J Med 2008;358:140-51.

9 Davies DM, de Vries PJ, Johnson SR, et al. Sirolimus therapy for angiomyolipoma in tuberous sclerosis and sporadic lymphangioleiomyomatosis: a phase 2 trial. Clin Cancer Res 2011;17:4071-81.

10 Bissler JJ, Kingswood JC, Radzikowska E, et al. Everolimus for angiomyolipoma associated with tuberous sclerosis complex or sporadic lymphangioleiomyomatosis (EXIST-2): a multicentre, randomised, double-blind, placebo-controlled trial. Lancet 2013;381:817-24.

11 Taveira-DaSilva AM, Hathaway O, Stylianou M, et al. Changes in lung function and chylous effusions in patients with lymphangioleiomyomatosis treated with sirolimus. Ann Intern Med 2011:154:797-805.

12 McCormack FX, Gupta N, Finlay GR, et al. Official American thoracic society/Japanese respiratory society clinical practice guidelines: lymphangioleiomyomatosis diagnosis and management. Am J Respir Crit Care Med 2016;194:748-61.

13 Krueger DA, Northrup H. International Tuberous Sclerosis Complex Consensus Group. Tuberous sclerosis complex surveillance and management: recommendations of the 2012 International Tuberous Sclerosis Complex Consensus Conference. Pediatr Neurol 2013:49:255-65.

14 Yao J, Taveira-DaSilva AM, Jones AM, et al. Sustained effects of sirolimus on lung function and cystic lung lesions in lymphangioleiomyomatosis. Am J Respir Crit Care Med 2014;190:1273-82.

15 Bee J, Bhatt R, McCafferty I, et al. A 4-year prospective evaluation of protocols to improve clinical outcomes for patients with lymphangioleiomyomatosis in a national clinical centre. Thorax 2015;70:1202-4.

16 Sasongko TH, Ismail NF, Zabidi-Hussin Z. Rapamycin and rapalogs for tuberous sclerosis complex. Cochrane Database Syst Rev 2016;7:CD011272.

17 Miller MR, Crapo R, Hankinson J, et al. General considerations for lung function testing. Eur Respir J 2005;26:153-61.

18 Quanjer PH, Tammeling GJ, Cotes JE, et al. Lung volumes and forced ventilatory flows. Report Working Party Standardization of Lung Function Tests, European Community for Steel and Coal. Official Statement of the European Respiratory Society. Eur Respir J Supp/ 1993;16:5-40.

19 Johnson SR, Tattersfield AE. Decline in lung function in lymphangioleiomyomatosis: relation to menopause and progesterone treatment. Am J Respir Crit Care Med 1999;160:628-33.

20 D'Armiento J, Shiomi T, Marks S, et al. Mesenchymal tumorigenesis driven by TSC2 haploinsufficiency requires HMGA2 and is independent of mTOR pathway activation. Cancer Res 2016:76:844-54.

21 Young L, Lee HS, Inoue Y, et al. Serum VEGF-D a concentration as a biomarker of lymphangioleiomyomatosis severity and treatment response: a prospective analysis of the Multicenter International Lymphangioleiomyomatosis Efficacy of Sirolimus (MILES) trial. Lancet Respir Med 2013:1:445-52.

22 Noh WC, Mondesire WH, Peng J, et al. Determinants of rapamycin sensitivity in breast cancer cells. Clin Cancer Res 2004:10:1013-23.

23 Sabatini DM. mTOR and cancer: insights into a complex relationship. Nat Rev Cancer 2006:6:729-34.

24 Kurmasheva RT, Huang S, Houghton PJ. Predicted mechanisms of resistance to mTOR inhibitors. Br J Cancer 2006;95:955-60.

25 Clements D, Dongre A, Krymskaya VP, et al. Wild type mesenchymal cells contribute to the lung pathology of lymphangioleiomyomatosis. PLoS One 2015;10:e0126025.

26 Ando K, Kurihara M, Kataoka H, et al. Efficacy and safety of low-dose sirolimus for treatment of lymphangioleiomyomatosis. Respir Investig 2013;51:175-83. 